\title{
Characteristics of structured physical training currently provided in cardiac patients: insights from the Exercise Training in Cardiac Rehabilitation (ETCR) Italian survey
}

\author{
Marco Ambrosetti', Patrick Doherty², Pompilio Faggiano33, Ugo Corrà4, Carlo Vigorito5, \\ Dominique Hansen6, Patrizio Sarto7, Ana Abreu8, Roberto F.E. Pedretti9
}

1 Cardiovascular Rehabilitation Unit, Le Terrazze Clinic, Cunardo (VA), Italy

2 Department of Health Sciences, University of York, UK

3 Department of Cardiology, University of Brescia, Italy

4 Department of Cardiac Rehabilitation, Maugeri Clinical Institutes, Veruno (NO), Italy

5 Department of Translational Medical Sciences, University of Naples Federico II, Naples

6 REVAL Rehabilitation Research Centre, BIOMED Biomedical Research Centre, Faculty of Medicine and Life Sciences, Hasselt University, Diepenbeek, Belgium

7 Department of Sports Medicine, Local Health Unit 9, Treviso, Italy

8 Department of Cardiology, Hospital Santa Marta, Lisbon, Portugal

9 Maugeri Clinical Institutes, Pavia, Italy

\begin{abstract}
Uncertainty exists about current delivery levels of exercise training (ET) during Cardiac Rehabilitation (CR) programmes. The aim of this study was to evaluate ET modalities in the real world of CR facilities in Italy. This was an observational survey of aggregate data, collected from CR facilities on a voluntary basis. Snapshots of a single working day at a local site were made, in terms of characteristics of patients and ET programmes delivered. Overall, 612 patients from 26 CR units were included, with an in-patient $v s$ out-patient ratio of 3:1. Coronary artery disease (57.6\%), heart failure (20.3\%), and valve disease/surgery (22.1\%) were the most represented target groups. The prevalence of endurance continuous training, interval training, and resistance/strength training was $66.7 \%, 11.1 \%$, and $9.0 \%$; other non-aerobic endurance and non-resistance training modalities such as respiratory muscle training and calisthenics were reported in $39.9 \%$ and $42.9 \%$ of cases respectively.
\end{abstract}

Corresponding author: Marco Ambrosetti, Cardiovascular Rehabilitation Unit, Le Terrazze Clinic, Via U. Foscolo 6/b, 21035 Cunardo (VA), Italy. Tel. +39.0332.992448. E-mail: m.ambrosetti@clinicaleterrazze.com

Key words: Exercise; cardiac rehabilitation; survey.

Received for publication: 20 September 2016

Accepted for publication: 17 October 2016

CCopyright M. Ambrosetti et al., 2017

Tipografia PI-ME Editrice, Italy

Monaldi Archives for Chest Disease 2017; 87:778

doi: $10.4081 /$ monaldi.2017.778

This article is distributed under the terms of the Creative Commons Attribution Noncommercial License (by-nc 4.0) which permits any noncommercial use, distribution, and reproduction in any medium, provided the original author(s) and source are credited.
Workloads for endurance exercise training were determined by cardiopulmonary test, conventional 12-leads ECG exercise testing, 6minwalking test, theoretical determination of heart rate, and rating of perceived exertion in $9 \%, 8 \%, 27 \%, 9 \%$, and $40 \%$ of cases respectively. The average duration of the programmes (on an intention to treat basis) was 25 sessions of $42 \pm 11 \mathrm{~min}$, with a frequency of $>4$ sessions/week in $67 \%$ of patients. Despite advances in CR interventions, there is a significant need for improvement of functional evaluation and exercise training prescription, and consideration of a wider range of training modalities in Italy.

\section{Introduction}

Exercise-based Cardiac Rehabilitation (CR) programmes have been recognized as appropriate and cost-effectiveness pathways aimed at decreasing hospital admissions, improving exercise capacity, conferring benefits in health-related quality of life, and reducing mortality in a variety of cardiac conditions [1-4]. The underutilization of CR programmes in several European countries [5] appears to be a significant problem and several barriers at the patient, provider, healthcare system, and community levels [6] may account for it. Among strategies to overcome this situation, educational efforts both aimed at the public and other healthcare providers play a pivotal role, by increasing the awareness of importance and benefits of CR. Nevertheless, despite the release of several performance measures and standards for intervention $[7,8]$, the often-reported heterogeneity of programmes [9] could question the ability of CR services to deliver all recognized core components of exercise training and secondary prevention, leading to a supposed attenuated effect of CR in the modern era of advanced revascularization and cardioprotective therapies. As a consequence, there is urgent need to evaluate the extent by which core CR domains (i.e., patient assessment, physical training, lifestyle modification, and psychosocial management) are being delivered in the real world.

Exercise training (ET) plays a fundamental role as a core component of $\mathrm{CR}$, whose modalities of prescription, supervision, and evaluation are nowadays well established [1]. Unfortunately, information about 
the current delivery of ET is poor and often limited to a generic assessment about the presence of such interventions within the delivery of CR services, as shown in the recent European overview of CR for patients with heart failure [10]. Even more, some prevention programmes, including patients' advice for physical activity and exercise reinforcement, leave out and disregard the importance of prescribed, structured, supervised exercise programmes. When available, information about ET among different target groups of CR generally lacks detail on drivers for prescription (i.e., frequency, intensity, type of exercise, duration).

The present study aimed to evaluate the extent of current modalities of ET prescription and management at a national level. Such a study is important, as it will establish the extent by which routinely delivery of CR aligns with of the published core components of modern ET prescription and act as a catalyst for improvement.

\section{Materials and Methods}

Between July and September 2015, the Italian Association of Cardiac Prevention and Rehabilitation (GICR-IACPR) invited all national CR services to participate to the Exercise Training in Cardiac Rehabilitation (ETCR) Italian survey on a voluntary basis. The call-to-participate was realized by posting a brief announcement on the GICR-IACPR website followed by two subsequent recalling newsletters. Centres were invited to choose a single working day (from Monday to Friday) and report aggregate data on patients fitting selected characteristics, by using a Categorization Form for Aggregate Data (CFAD). Since the study was based on anonymized patient data within a predetermined model of categorization, no request to the Ethics Committee was applied. Data governance procedures adhered to the policies of the GICRIACPR Study Center for Research and Education. The CFAD was built and validated by expert-opinion as part of a collaborative project between GICR-IACPR and the Nucleus of Cardiac Rehabilitation Section of the European Association of Cardiovascular Prevention and Rehabilitation (NCR-EACPR), and the first wave of the survey within the Italian network served as a test for further utilization of the tool among other European countries.

The CFAD focused on patients with an ongoing exercise programme during the snapshot day and collected data on type of exercise, presence of supervision, setting, modality of exercise prescription, and workload (exercise intensity) according to different patient groups: coronary artery disease (CAD), chronic heart failure (CHF), valve disease/valvular surgery (VDS), device (implantable cardiac defibrillator, cardiac resynchronization therapy, or left ventricular assist device) and/or cardiac transplantation recipients, peripheral arterial disease, congenital heart disease, and pulmonary hypertension. In case of combined disease, only the index condition for referral to CR was considered. Information about frequency of training sessions, duration of training sessions, and total programme duration was based on an intention to treat approach.

Descriptors of ET were derived from EACPR position papers [11,12]. Endurance continuous training (ECT) was defined as an activity supported by aerobic metabolism that engages large muscle groups in a rhythmical manner, but at a continuous intensity. Interval training (IT) described training programmes during which the patient was asked to alternate short bouts of moderate-to-high intensity exercise, with a longer recovery phase in between, performed at low or no workload. Resistance/strength training (RST) was characterized by series of muscle contractions performed against resistance that was sufficient to overload the musculoskeletal system. Respiratory muscle training (RT) included any different protocol or device for inspiratory muscle training (i.e., isocapnic hyperpnoea, incentive spirometry, or resistive pressure threshold load), while Calisthenics (CT) described a variety of exercises (often rhythmical movements and stretches included) generally intended to increase musculoskeletal flexibility and agility.

The following modalities for workload or exercise intensity assessment were identified: 1) determination of oxygen consumption by cardiopulmonary exercise testing (CPET) with gas exchange analysis, 2) conventional 12-leads ECG exercise testing (SET) for the determination of target heart rates, 3) Ratings of Perceived Exertion (RPE) (i.e., traditional Borg or modified CR10 Borg scales), 3) Six minutes walking test (6MWT), 4) predicted exercise-related heart rates or energy expenditures without exercise testing, and 5) other indirect evaluations (i.e., the 'walk test' or 'walk and talk test') [12].

The following chronic cardiovascular and non-cardiovascular conditions were reported as they have a known role in modulating exercise prescription in the context of CR: obesity (BMI $>30 \mathrm{~kg} / \mathrm{m}^{2}$ ), arterial hypertension ( $\mathrm{SBP} \geq 140 \mathrm{mmHg}$ or $\mathrm{DBP} \geq 90 \mathrm{mmHg}$ ), dyslipidemia [defined as elevated total or low-density lipoprotein (LDL) cholesterol levels, or low levels of high-density lipoprotein (HDL) cholesterol], diabetes mellitus (HbAlc>6.4\%), smoking, depression, pacemaker /implantable cardioverter defibrillator/ cardiac resynchronisation therapy, pulmonary arterial hypertension, chronic lung disease (e.g., chronic obstructive pulmonary disease, interstitial lung disease, etc.), severe renal failure (GFR $\leq 30 \mathrm{~mL} / \mathrm{min} / 1.73 \mathrm{~m}^{2}$ ), sarcopenia/frailty (defined as progressive and generalized loss of skeletal muscle mass and strength, and a status of vulnerability characterized by declining function of multiple physiologic systems respectively), and elderly ( $>75$ years-old).

The in-patient setting referred to CR programmes that required full admission for the day and overnight, while in the out-patient setting treatments were provided on an ambulatory basis or through day hospital arrangement.

\section{Sample size calculation}

The estimated minimum number of sites to produce representative survey results was based on previous GICR-IACPR national registries [13]. We determined that to achieve the 95\% confidence interval around the estimate of $98.4 \%$ (i.e., the provision rate of ET activities reported by all 190 Italian CR facilities) with a 7\% absolute precision, a minimum of 15 institutions should have been targeted.

\section{Statistical analysis}

All responses collected via the GICR-IACPR website were inspected, downloaded and securely stored by the GICR-IACPR Study Center for Research and Education. Results were shown as mean (SD) or as proportions, and comparisons were by t test (for interval data with normal distribution), $\chi^{2}$ test (for nominal data) or Mann-Witney test (for interval data with skewed distribution) as appropriate. The SPSS package (SPSS Inc. Released 2008. SPSS Statistics for Windows, Version 17.0. Chicago: SPSS Inc) was used to perform statistical analysis. Statistical significance was set at $\mathrm{p}<0.05$ (two-tailed).

\section{Results}

Twenty-six 26 CR services collected data on 612 patients ( $25 \pm 17$ patients per unit). The average number of ET programmes delivered per unit/year was $444 \pm 344$, and $592(96.7 \%)$ patients were actively engaged into ET activities during the snapshot day. 
Overall, 458 (77.4\%) and 134 (22.6\%) participants were classified as in-patients and out-patients respectively, and supervision of ET was provided in 550 subjects ( $92.9 \%)$.

Patients with CAD ( $\mathrm{n}=323 ; 57.6 \%)$, CHF ( $\mathrm{n}=114 ; 20.3 \%)$, and VDS ( $\mathrm{n}=124 ; 22.1 \%$ ) accounted for $95 \%$ of the ET population and represent the focus of this paper. Endurance continuous training (ECT) was the most represented type of exercise, with an implementation rate of $66.7 \%(\mathrm{n}=396)$, while IT, RST, RT, and CT protocols were implemented in 11.1\% $(\mathrm{n}=66), 9.0 \%(\mathrm{n}=53), 39.9 \%(\mathrm{n}=$ $236)$, and $42.9 \%(\mathrm{n}=254)$ of patients respectively. Among the three major target groups (Figure 1), CAD patients displayed the highest rate of implementation of ECT (73.1\%), while greatest implementation rates of IT and RST were documented among CHF (14.0\% and $13.2 \%$ respectively); VDS patients showed greatest implementation rate of RT (62.9\%). The ECT programme was more frequently implemented among out-patients as compared to in-patients $(85.1 \% \mathrm{vs}$ $61.6 \%, \mathrm{p}<0.001)$, while in-patients were more frequently engaged into RT $(44.5 \%$ vs $23.9 \%, \mathrm{p}<0.001)$ and CT $(52.2 \%$ vs $40.2 \%, \mathrm{p}<0.05)$ protocols; IT (13.4\% vs $10.5 \%, \mathrm{p}=0.460)$ and RST implementation rates $(9.2 \%$ vs $8.2 \%, \mathrm{p}=0.854)$ did not significantly differ between these two settings.

Globally, exercise or workload intensity determination for endurance exercise training by means of CPET did not exceed 9\% within the studied patients (Figure 2). Similarly, the adoption of SET for exercise or workload intensity determination was about $8 \%$, and the most utilized tool for this determination was represented by RPE (40\%) guiding exercise without a baseline exercise test. The utilization of 6MWT and $\mathrm{RPE}$, as a driver for exercise intensity prescription, was more frequently used among in-patients (Figure 3), while CPET and SET was more often used in the out-patient setting.
Among patients performing aerobic exercise training, a light to moderate-intensity domain was reported in $45.1 \%$ of the cases (Figure 4), while moderate-to-high- and high-to-severe-intensity domains were present in $53.1 \%$ and $1.8 \%$, respectively, of the cases. A moderate-to-high-intensity exercise training protocol was performed more frequently in CAD patients, as compared to CHF and VDS patients $(62.4 \%$ vs $29.5 \%$, or $47.5 \%, \mathrm{p}<0.01$ respectively), with a significant split across out-patients compared to in-patients $(82.6 \%$ vs $42.9 \%, \mathrm{p}<0.001)$.

The number of training sessions during the CR programme ranged from $12 \pm 6$ for out-patients with CHF and preserved ejection fraction to $26 \pm 9$ for out-patients with $\mathrm{CAD}$, with an average of $25 \pm 6$ in the whole population. In summary, $66.7 \%$ of patients were trained at least 5 days per week. The duration of each session was $42 \pm 11$ minutes. The prevalence of potential modulating factors for the exercise programme is shown in Figure 5.

\section{Discussion}

The ETCR Italian survey was designed to take a snapshot of exercise training activities currently performed in the real world of CR services. Such snapshot reflects how exercise training is delivered and prescribed over a longer period to cardiac patients, as most CR centers do not change their exercise prescription and implantation frequently.

Despite ET being a major core component of CR programmes [7], several observational studies examining the provision of CR activities [10,14-17] have found that cardiac patients may be receiving less than optimal exercise training prescriptions, with substantial variability between facilities and obscurities about exercise training prescription methods.

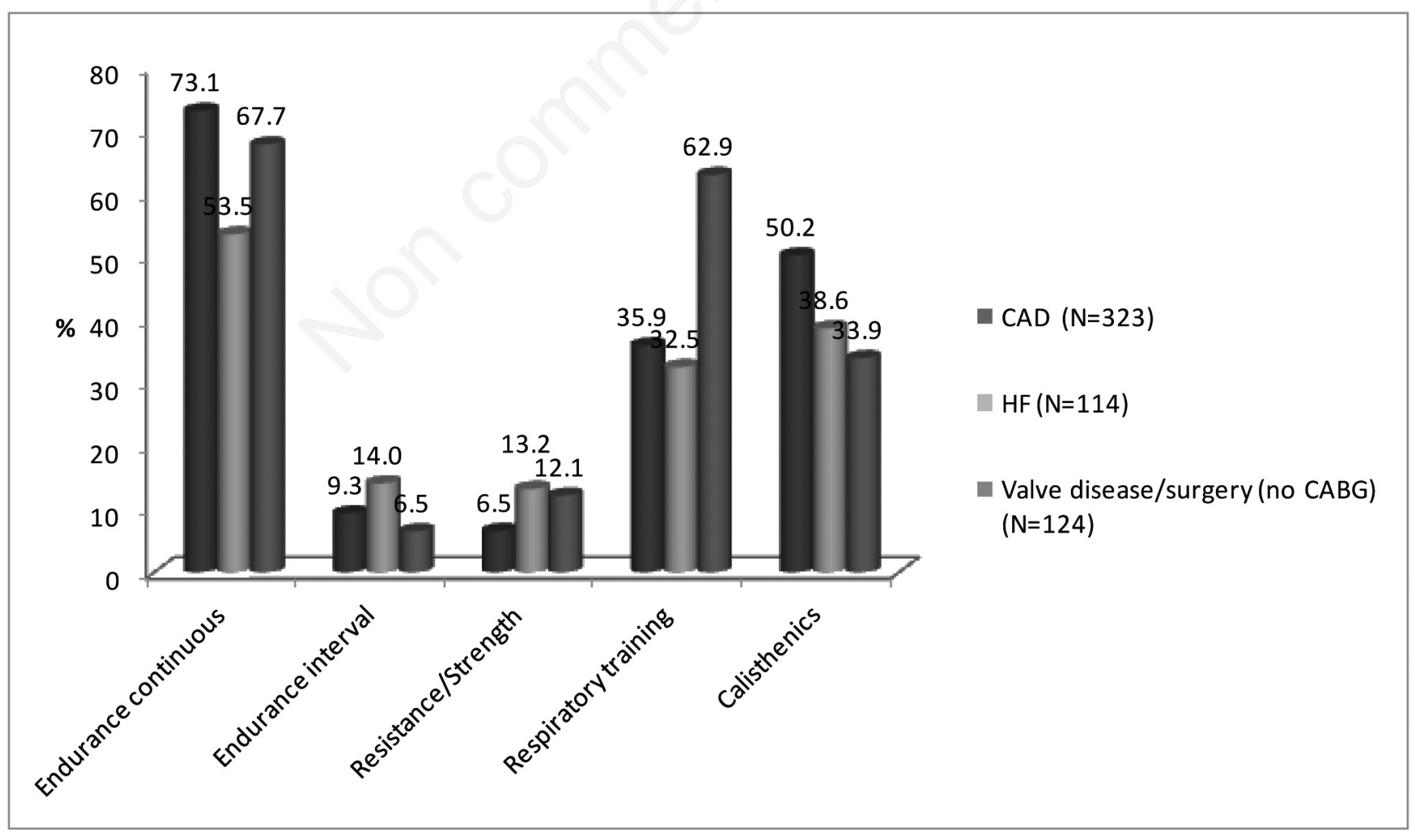

Figure 1. Types of exercise training in coronary (CAD), heart failure (HF) and valve disease/surgery patients with an ongoing Cardiac Rehabilitation program. 


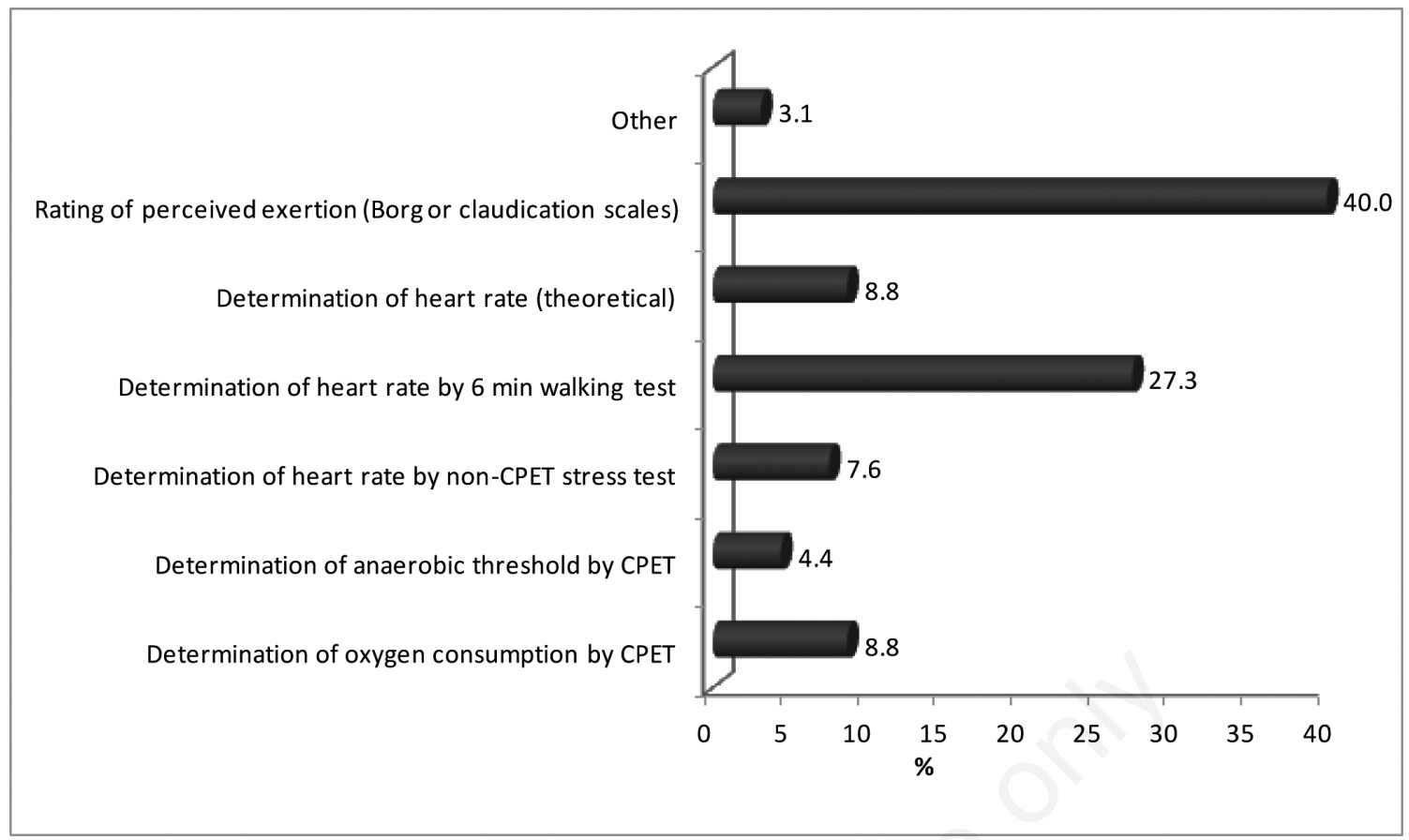

Figure 2. Prevalence of different methods to evaluate workloads for endurance in patients with an ongoing Cardiac Rehabilitation programme $(\mathrm{n}=592)$.

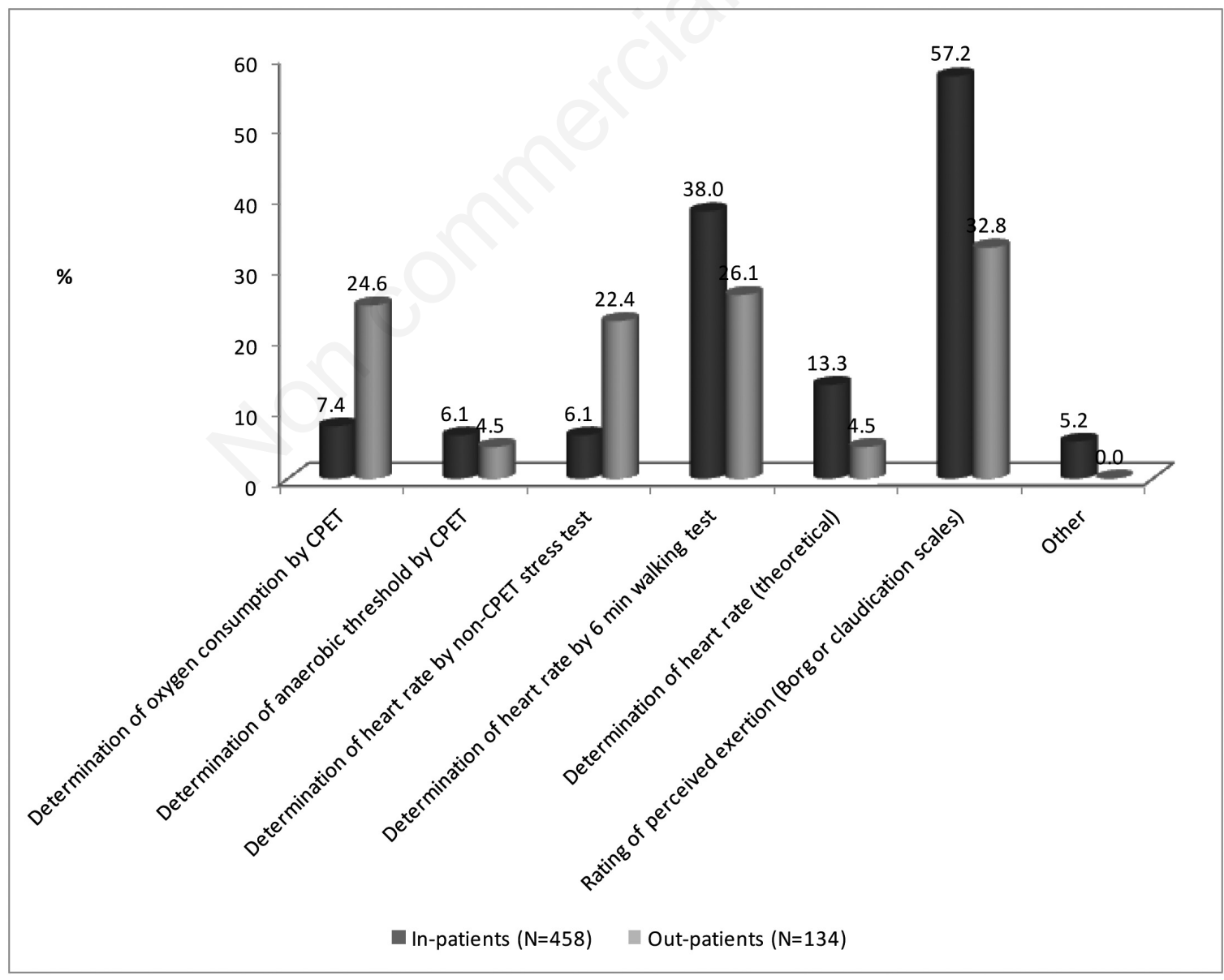

Figure 3. Prevalence of different methods to evaluate workloads for endurance by setting $(\mathbf{n}=592)$. 


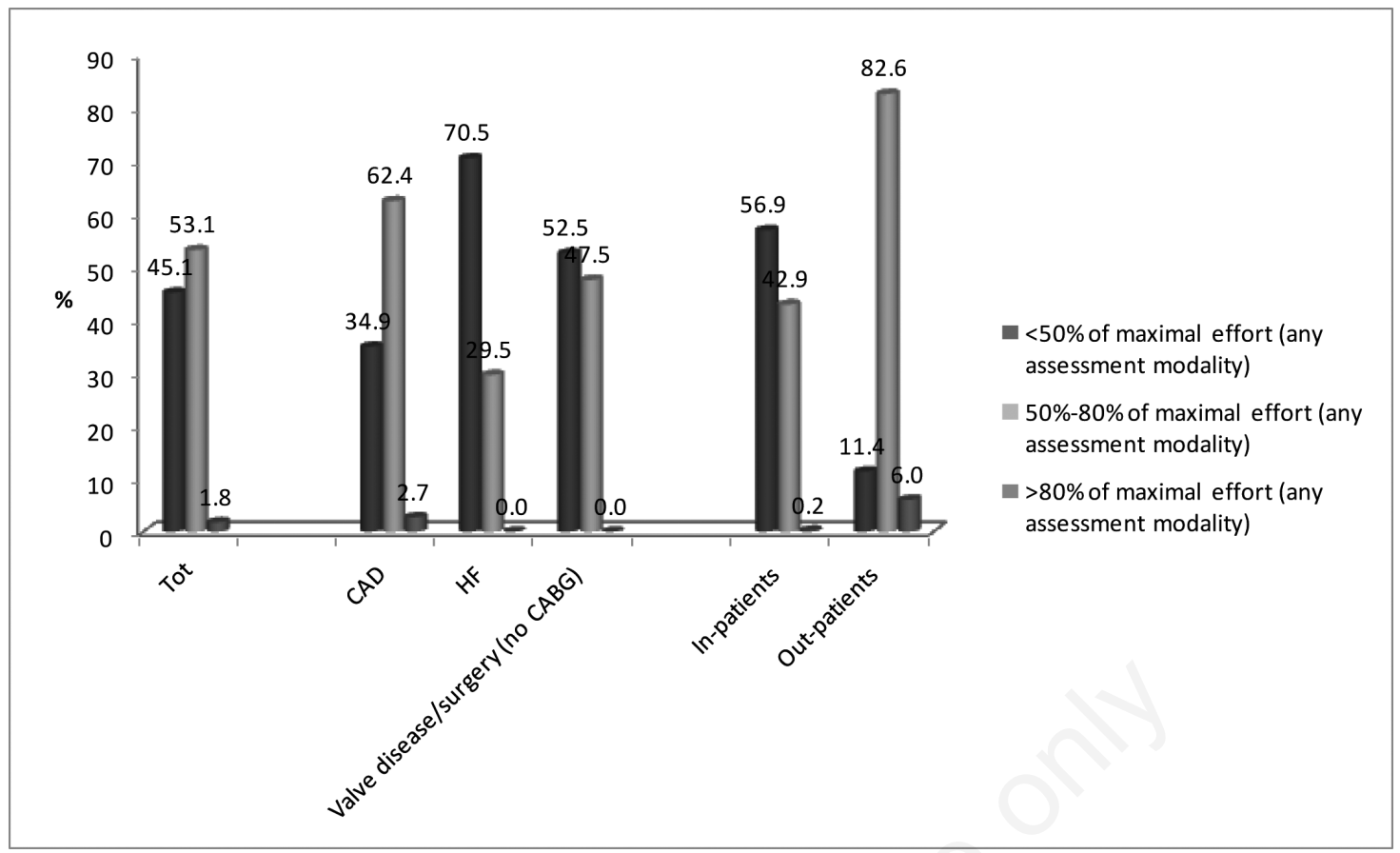

Figure 4. Intensity domains of endurance training $(n=592)$.

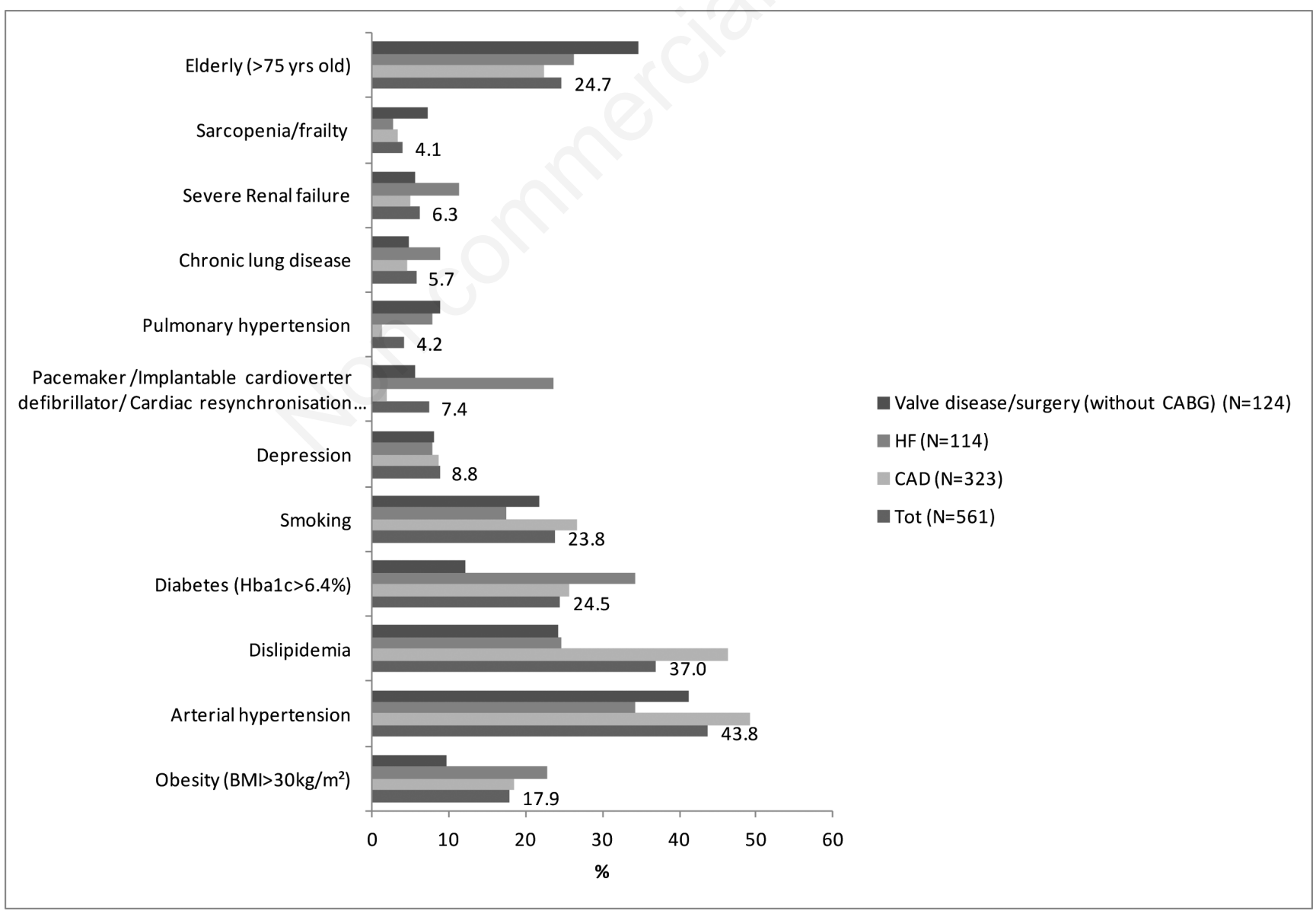

Figure 5. Prevalence of factors potentially modulating exercise prescription in the whole population and major subgroups. 
Notably, data from CR centres concerning functional capacity assessment methodology, and preferred type, duration, frequency, and intensity of exercise training, are sparse. Notwithstanding the established importance of CR in cardiac patients, some observations were made in the present study that may be of concern and should deserve greater attention and/or adjustment in the near future to optimize the clinical effectiveness, exercise training adherence, and/or medical safety of CR programmes.

Our study showed that no more than two-thirds of CR patients usually receive ECT in daily practice. This is below the recommended level and inconsistent with current evidence which highlights the beneficial effects of ECT on symptom-free exercise capacity, quality of life, and life expectancy [1]. At first glance, fewer rates of ECT may reflect a problem of non-adherence, since CR participants usually attend at 66.5 $\pm 18.2 \%$ of the prescribed exercise training sessions, as reported by a recent meta-analysis [18]. Most probably, this finding may also be related to the high complexity and relatively fewer clinically stable patients currently referred to CR programmes in Italy, due to short hospitalization in acute wards, elevated age, and marked disability. In several circumstances patients may be too heavily compromised to perform ECT even at low workload, and consequently need their CR programmes to be based on other non-aerobic endurance and non-resistance training modalities. The high rate of RT and CT we found (about 40\%) may emphasize the role of respiratory intervention and light exercises as the only permitted training activity in such cases. This explanation was also supported by higher rates of ECT and higher intensity activities found for out of the hospital setting, where clinical conditions are assumed to be more stable.

Similar considerations may also apply for the low use of IT and RST in CR, whose requirements in terms of clinical stabilization and exclusion criteria due to safety reasons are even more stringent as compared to ECT [19], thus limiting the proportion of eligible patients recruited. IT and RST constitute the most recent forms of ET available to patients with cardiac disease and, whereas providing clinical improvements in terms of cardiovascular fitness and strength [20-22], their incorporation into standard CR programmes - either as alternative or as an adjunct to ECT - has not been concretely reached yet. It was also important to note the higher use of IT and RST protocols among CHF as compared to CAD patients in our study, despite the predominant body of evidence for the latter patient group, probably due to the availability of advanced pathways for such patients.

A critical point is given by the substantial low number of patients (i.e. less than $20 \%$ out of all CR participants in our study) who receive structured exercise testing to determine training intensity and volume. Data on the provision of exercise testing during CR on a patient population basis are sparse, since this estimate was generally obtained on a facility level, with marked discrepancies between countries: Bradley et al. showed that only $12.5 \%$ of CR centres used exercise testing on a regular basis in Northern Ireland [23], while in the New York State [24] and in Australia [25] this percentage elevated up to $90 \%$.

Current guidelines [12] clearly recommend that exercise testing is an essential tool for exercise training evaluation, risk stratification to determine the required level of supervision and monitoring, and individualized exercise prescription. Particularly in the case of CHF, CPET is specifically indicated, however in the present study less than $10 \%$ of CHF patients attending CR had this testing performed. Although it is perhaps challenging to offer a formal exercise testing before initiating ET in all phase II CR patients, especially if fast-tracked to rehabilitative facilities, it is remarkable that the evaluation of peak exercise capacity by means of SET and/or CPET was so low. Baseline assessment of exercise capacity still remains a fundamental process-based performance measure [7] for clinical care in CR.
Aerobic training in the moderate-to-high-intensity domain is supported by strong evidence in cardiac conditions with both preserved and reduced left ventricular function [26,27], nevertheless just half of patients were prescribed such training intensity in our survey. Probable reasons, as safety and supervision concerns and lack of CPET or even conventional exercise testing performance, may be the basis of the use of lower exercise training intensities. This practice, however, may lead to suboptimal exercise training effects, as evidenced by smaller improvements in endurance exercise capacity, but also by smaller effects on certain cardiovascular risk factors (such as blood lipid profile and adipose tissue mass). In a recent German monocentric cohort [28], the majority of CR patients underwent training sessions with moderate to high-intensity and reached a considerable increase of their training volume, thus indicating that more sustained ET could be offered on a large scale in CR practice, at least for those patients at low risk and less advanced age.

Our study has limitations. Firstly, data aggregation did not enable us to evaluate the association between ET activities and clinical characteristics of the enrolled population, as far as the number of patients taking part in multi-modality exercise types. For these purposes the implementation of an observational case series study would be the most appropriate choice. On the other hand, the utilization of a CFAD for a single-day survey, though less informative, could improve the rate of participating facilities and allow multiwave snapshots to capture changes. Secondly, information was collected within the Italian health system, and results probably can be generalized to similar environments only (i.e., presence of financial provision, CR facilities often linked to dedicated cardiology division, predominance of residential CR programs, structural heterogeneity of services, and availability of national guidelines [13]). Finally, information was collected on a limited subset (about 15\%) of existing facilities in Italy [13].

In conclusion, the ETCR Italian survey pointed out several critical issues in the actual provision of exercise-based CR programs in Italy, namely the limited adoption of traditional and newer modalities of training, the underuse of CPET and SET for the assessment of functional capacity, and the preferential prescription of low-intensity exercise. As a consequence, efforts aimed at increasing the number of patients included in CR programmes strongly need to be supported by further elevation of performances and quality of care. National and European societies need to be engaged in the implementation of CR minimal standards compliance, CR health professionals' education and certification, and internal and external audits, in order to improve quality and consequent benefits of CR programmes.

\section{References}

1. Anderson L, Thompson DR, Oldridge N, et al. Exercise-based cardiac rehabilitation for coronary heart disease. Cochrane Database Syst Rev 2016;1:CD001800.

2. Taylor RS, Sagar VA, Davies EJ, et al. Exercise-based rehabilitation for heart failure. Cochrane Database Syst Rev 2014;4:CD003331.

3. Sibilitz KL, Berg SK, Tang LH, et al. Exercise-based cardiac rehabilitation for adults after heart valve surgery. Cochrane Database Syst Rev 2016;3:CD010876.

4. Wong WP, Feng J, Pwee KH, Lim J. A systematic review of economic evaluations of cardiac rehabilitation. BMC Health Serv Res 2012; 12:243.

5. Clark AM, King-Shier KM, Duncan A, et al. Factors influencing referral to cardiac rehabilitation and secondary prevention programs: a systematic review. Eur J Prev Cardiol 2013;20:692-700. 
6. Thomas RJ. Cardiac Rehabilitation/Secondary Prevention Programs. A raft for the rapids: why have we missed the boat? Circulation 2007;116:1644-6.

7. Piepoli MF, Corrà U, Adamopoulos $\mathrm{S}$, et al. Secondary prevention in the clinical management of patients with cardiovascular diseases. Core components, standards and outcome measures for referral and delivery: a policy statement from the cardiac rehabilitation section of the European Association for Cardiovascular Prevention and Rehabilitation. Endorsed by the Committee for Practice Guidelines of the European Society of Cardiology. Eur J Prev Cardiol 2014; 21:664-81.

8. Thomas RJ, King M, Lui K, et al. AACVPR/ACCF/AHA 2010 Update: Performance measures on cardiac rehabilitation for referral to cardiac rehabilitation/secondary prevention services: A report of the American Association of Cardiovascular and Pulmonary Rehabilitation and the American College of Cardiology Foundation/American Heart Association Task Force on Performance Measures (Writing Committee to Develop Clinical Performance Measures for Cardiac Rehabilitation). J Cardiopulm Rehabil Prev 2010;30:279-88.

9. West RR, Jones DA, Henderson AH. Rehabilitation after myocardial infarction trial (RAMIT): multi-centre randomised controlled trial of comprehensive cardiac rehabilitation in patients following acute myocardial infarction. Heart 2012;98:637-44.

10. Piepoli MF, Binno S, Corrà U, et al. ExtraHF survey: the first European survey on implementation of exercise training in heart failure patients. Eur J Heart Fail 2015;17:631-8.

11. Piepoli MF, Corra` U, Benzer W, et al. Secondary prevention through cardiac rehabilitation: physical activity counselling and exercise training. Eur Heart J 2010;31:1967-76.

12. Mezzani A, Hamm LF, Jones AM, et al. Aerobic exercise intensity assessment and prescription in cardiac rehabilitation: a joint position statement of the European Association for Cardiovascular Prevention and Rehabilitation, the American Association of Cardiovascular and Pulmonary Rehabilitation and the Canadian Association of Cardiac Rehabilitation. Eur J Prev Cardiol 2012;20:442-67.

13. Tramarin R, Ambrosetti M, De Feo S, et al. The Italian Survey on Cardiac Rehabilitation-2008 (ISYDE-2008). Part 3. National availability and organization of cardiac rehabilitation facilities. Official report of the Italian Association for Cardiovascular Prevention, Rehabilitation and Epidemiology (IACPR-GICR). Monaldi Arch Chest Dis 2008;70:175-205.

14. Bjarnason-Wehrens B, McGee H, Zwisler AD, et al. Cardiac rehabilitation in Europe: results from the European Cardiac Rehabilitation Inventory Survey. Eur J Cardiovasc Prev Rehabil 2010;17:410-8.

15. Vromen T, Spee RF, Kraal JJ, et al. Exercise training programs in Dutch cardiac rehabilitation centres. Netherlands Hear J 2013;21:138-43.

16. Brodie D, Bethell H, Breen S. Cardiac rehabilitation in England: a detailed national survey. Eur J Cardiovasc Prev Rehabil 2006;13: $122-8$.

17. McGee HM, Hevey D, Horgan JH. Cardiac rehabilitation service provision in Ireland: the Irish Association of Cardiac Rehabilitation survey. Ir J Med Sci 1998;170:159-62.

18. Oosenbrug E, Marinho RP, Zhang J, et al. Sex differences in cardiac rehabilitation adherence: a meta-analysis. Can J Cardiol 2016;32: 1316-24.
19. Bjarnason-Wehrensa B, Mayer-Bergerb W, Meisterc ER, et al. Recommendations for resistance exercise in cardiac rehabilitation. Recommendations of the German Federation for Cardiovascular Prevention and Rehabilitation. Eur J Cardiovasc Prev Rehabil 2004; 11:352-61.

20. Guiraud T, Nigam A, Gremeaux V, et al. High-intensity interval training in cardiac rehabilitation. Sports Med 2012;42:587-605.

21. Gayda M, Ribeiro PA, Juneau M, et al. Comparison of different forms of exercise training in patients with cardiac disease: where does high-intensity interval training fit? Can J Cardiol 2016; 32: 485-94.

22. Marzolini S, Oh PI, Brooks D. Effect of combined aerobic and resistance training versus aerobic training alone in individuals with coronary artery disease: a meta-analysis. Eur J Prev Cardiol 2011; 19:81-94.

23. Bradley JM, Wallace ES, McCoy PM, et al. A survey of exercise based cardiac rehabilitation services in Northern Ireland. Ulster Med J 1997;66:100-6.

24. Deturk WE, Scott LB. Physical therapists as providers of care: exercise prescriptions and resultant outcomes in cardiac and pulmonary rehabilitation programs in New York State. Cardiopulm Phys Ther J 2008;19:35-43.

25. Abell B, Glasziou P, Briffa T, et al. Exercise training characteristics in cardiac rehabilitation programmes: a cross-sectional survey of Australian practice. Open Heart 2016;3:e000374.

26. Adachi H, Koike A, Obayashi T, et al. Does appropriate endurance exercise training improve cardiac function in patients with prior myocardial infarction? Eur Heart J 1996;17:1511-21.

27. Dubach P, Myers J, Dziekan G, et al. Effect of high intensity exercise training on central hemodynamic responses to exercise in men with reduced left ventricular function. J Am Coll Cardiol 1997; 29:1591-8.

28. Reibis R, Salzwedel A, Buhlert H, et al. Impact of training methods and patient characteristics on exercise capacity in patients in cardiovascular rehabilitation. Eur J Prev Cardiol 2016;23:452-9.

\section{ETCR Study Group}

B. Passaretti (Bergamo), E. Venturini (Cecina), R. Carlon (Cittadella), G. Vitali (Cuggiono), M. Ambrosetti (Cunardo), F. Fattirolli (Firenze), A. Molteni (Lugano), 0. Tortelli (Mantova), A. Longoni (Mariano Comense), S. Riccobono (Milano), 0. Febo (Montescano), C. Vigorito (Napoli), M. Palvarini (Passirana), M. Perotti (Pavia), A. Rosi (Ponte dell'Olio), B. Miserrafiti (Melito Porto Salvo), 0. Febo (Rivolta d'Adda), M. Ruzzolini (Roma), S. Aglieri (Rozzano), M. Persico (San Benedetto del Tronto), D. Bertoli (Sarzana), S. Sarzi Braga (Tradate), M. Werren (Udine), R. Reggiani (Verano Brianza), M. Provvidenza (Lido di Camaiore), M. Pistono (Veruno).

\section{CFAD validation Expert Group}

A. Abreu (P), M. Ambrosetti (I), R. Belardinelli (I), U. Corrà (I), M. Cupples (UK), C. Davos (GR), P. Doherty (UK), F. Edelmann (D), P. Faggiano (I), E. Greco (I), D. Hansen (B), S. Hofer (A), MC Iliou (F), RFE. Pedretti (I), M. Piepoli (I), B. Rauch (D), JP Schmid (CH), C. Vigorito (I), H. Voller (D). 